\title{
Some new convergence and stability results for Jungck generalized pseudo-contractive and Lipschitzian type operators using hybrid iterative techniques in the Hilbert space
}

\author{
M. O. Olatinwo ${ }^{1}$ D. O. J. Omidire ${ }^{1}$
}

Received: 1 April 2021 / Accepted: 24 July 2021 / Published online: 29 January 2022

(C) The Author(s) 2022

\begin{abstract}
The convergence of generalized pseudo-contractive operators were studied with regard to a unique fixed point in the article (Verma in Proc Roy Irish Acad Soct A, 97(1):83-86, 1997). In this present article, we introduce Jungck generalized pseudo-contractive and Lipschitzian type operators in the prehilbertian space and in the Hilbert space settings, establish the existence and the uniqueness of a common fixed point for the operator $S$ and a sequence of operators $\left\{T_{i}: D \rightarrow D\right\}_{i=1}^{k}$ using a new Jungck-Kirk-Mann type fixed point iterative algorithm as well as the general Kirk-Mann type iterative algorithm. The strong convergence of these fixed point iterative algorithms to a unique common fixed point is also investigated for the same set of operators considered. Moreover, some stability results are established for the Jungck-Kirk-Mann type iterative algorithm as well as the general Kirk-Mann type iterative algorithm. In addition, some examples are given to support our arguments. The results are new, original as well as generalizing some existing ones in the literature.
\end{abstract}

Keywords Jungck generalized pseudo-contractive and Lipschtizian type operators . Generalized pseudo-contractive and Lipschitzian · Jungck-Kirk-Mann type iterative algorithm · General Kirk-Mann type $\cdot$ Unique common fixed point

Mathematics Subject Classification $47 \mathrm{H} 06 \cdot 54 \mathrm{H} 25$

M. O. Olatinwo

memudu.olatinwo@gmail.com; molaposi@yahoo.com; polatinwo@oauife.edu.ng

O. J. Omidire

omidireolaoluwa@gmail.com; omidireolaoluwa@yahoo.com

1 Department of Mathematics, Obafemi Awolowo University, Ile-Ife, Nigeria 


\section{Introduction}

Verma [42], it was proved that Schaefer's fixed point iterative process converges to the unique fixed point of a generalized pseudo-contractive and Lipschitzian operator in the Hilbert space. Nevertheless, to the best of our knowledge, convergence of an iterative process to the unique common fixed point of such operator has not been considered in the literature. Theorem 3.6 of Berinde [5] was the first of both convergence results proved in [42] (which is Theorem 2.1 in [42]). We refer to Browder and Petryshyn [7, 8] as well as the papers [43-46] for a detailed and germane study concerning convergence of iterative processes to fixed points of nonlinear mappings. The interested readers can also consult the recent articles in [6, 15, 27-29, 35-41] for an excellent study on the foregoing convergence notion.

Our purpose in this article is to examine the existence and the uniqueness of a common fixed point for the operator $S$ and a sequence of operators $\left\{T_{i}: D \rightarrow D\right\}_{i=1}^{k}$ using a new Jungck-Kirk-Mann type fixed point iterative algorithm as well as the general Kirk-Mann type iterative algorithm. The strong convergence of these fixed point iterative algorithms to a unique common fixed point is also investigated when each $T_{i}$ is Jungck generalized pseudo-contractive and Lipschitzian type and when each $T_{i}$ is generalized pseudo-contractive and Lipschitzian. The new iterative algorithm is also considered when each $T_{i}$ is Jungck generalized pseudo-contractive and nonexpansive type. We support our arguments concerning the new type of operators with some examples.

In addition, a new notion of stability is given in Sect. 4 to establish some stability results for the family of operators. Our results generalize and extend the existing ones in the literature. For an excellently detailed study on the stability of fixed point iterative algorithms and various contractive conditions, we refer to the articles $[9,24,25,30-32$, 34] and the papers of the author [18-20].

\section{Preliminaries}

The following definitions shall be required in the sequel:

Definition 2.1 Let $Y$ be a normed space, and $S, T: Y \rightarrow Y$. Then, $T$ is said to be Lipschitzian type if there exists $L>0$ such that

$$
\|T u-T v\| \leq L\|S u-S v\|, T(Y) \subset S(Y), \quad \forall u, v \in Y .
$$

\section{Remark 2.2}

(i) With $L=1, S=I=$ Identity operator, then $T$ becomes the classical nonexpansive mapping.

(ii) If $L \in[0,1)$, (1) reduces to the celebrated Jungck contractive condition. See [11].

(iii) If $L=1, T$ is called $S$-nonexpansive operator, and this is contained in Olatinwo [16]. Although, in the present article, an $S$-nonexpansive operator shall be called a nonexpansive type operator.

Also, we introduce a class of contractive operators in the Hilbert space setting satisfying the following contractive inequality condition: 
Definition 2.3 Let $H$ be a real prehilbertian space and $\langle.,$.$\rangle an inner product on H$. Also let $\|\cdot\|$ be the norm induced by the inner product. An operator $T: H \rightarrow H$ is said to be Jungck generalized pseudocontractive, or, a Jungck generalized pseudocontraction if there exist $r>0$ and an operator $S: H \rightarrow H$ such that $\forall u, v \in H$,

$$
\|T u-T v\|^{2} \leq r^{2}\|S u-S v\|^{2}+\|T u-T v-r(S u-S v)\|^{2}, T(H) \subset S(H) .
$$

Now, from (2) above, we have by using the fact that the norm is induced by the inner product that

$$
\begin{aligned}
\langle T u-T v, T u-T v\rangle \leq & r^{2}\langle S u-S v, S u-S v\rangle \\
& +\langle T u-T v-r(S u-S v), T u-T v-r(S u-S v)\rangle \\
= & r^{2}\langle S u-S v, S u-S v\rangle+\langle T u-T v-r(S u-S v), T u-T v\rangle \\
& +\langle T u-T v-r(S u-S v),-r(S u-S v)\rangle \\
= & r^{2}\langle S u-S v, S u-S v\rangle+\langle T u-T v, T u-T v\rangle \\
& +\langle-r(S u-S v), T u-T v\rangle \\
& +\langle T u-T v,-r(S u-S v)\rangle+\langle-r(S u-S v),-r(S u-S v)\rangle,
\end{aligned}
$$

which reduces to

$$
0 \leq 2 r^{2}\langle S u-S v, S u-S v\rangle-2 r\langle T u-T v, S u-S v\rangle
$$

that is,

$$
\langle T u-T v, S u-S v\rangle \leq r\|S u-S v\|^{2},
$$

thus, showing that Inequality condition (2) is equivalent to that in $(\Delta)$.

Also, subtracting each side of Inequality $(\Delta)$ from $\langle S u-S v, S u-S v\rangle$ and using the fact that the norm is induced by the inner product yield

$$
\langle S u-S v, S u-S v\rangle-\langle T u-T v, S u-S v\rangle \geq(1-r)\langle S u-S v, S u-S v\rangle,
$$

that is,

$$
\langle(S-T) u-(S-T) v, S u-S v\rangle \geq(1-r)\|S u-S v\|^{2},
$$

which proves that Inequality condition (2) is equivalent to that in $(\Delta \Delta)$ too.

Applying the Cauchy-Schwarz inequality in $(\Delta)$ yields

$$
\|T u-T v\| \cdot\|S u-S v\| \leq|r| \cdot\|S u-S v\|^{2},
$$

that is,

$$
\|T u-T v\| \leq r\|S u-S v\|, T(H) \subset S(H) .
$$




\section{Remark 2.4}

(i) In Inequality (3), if $r=L$, then $T$ becomes a Lipschitzian type operator. That is, any Lipschitzian type operator is also Jungck generalized pseudo-contractive.

(ii) If $r \in[0,1)$, then (3) becomes Jungck contraction (see [11]).

(iii) If in (3), $r=1, S=I=$ Identity operator, then, $T$ is a non-expansive operator.

(iv) If in (2), $S=I=$ Identity operator, then we have the class of generalized pseudocontractive operators. That is, a generalized pseudo-contractive operator $T$ satisfies

$$
\|T u-T v\|^{2} \leq r^{2}\|u-v\|^{2}+\|T u-T v-r(u-v)\|^{2}, \quad u, v \in H, \quad r>0 .
$$

See Berinde [3-5] and Verma [42] for Inequality (4) above.

(v) If in (4), $r=1$, then, we obtain the class of pseudo-contractive operators. This is contained in $[3-5,8,42]$ and a host of other references.

In a complete metric space, for two commuting operators $S, U: X \rightarrow X$ and for any $x_{0} \in X$, the sequence $\left\{S x_{n}\right\}_{n=0}^{\infty} \subset X$ defined by

$$
S x_{n+1}=U x_{n}, \quad n=0,1,2, \ldots,
$$

is the iterative process associated to the result of Jungck [11].

Lemma 2.5 (Banach's Fixed Point Theorem $[1,2,5])$ Let $(X, d)$ be a complete metric space and $T: X \rightarrow X$ a contraction, that is, an operator satisfying

$$
d(T x, T y) \leq \eta d(x, y), \quad \forall x, y \in X, \eta \in[0,1) .
$$

Then, Thas a unique fixed point in $X$.

Lemma 2.6 (Jungck's Common Fixed Point Theorem [11]) Let $(X, d)$ be a complete metric space and $S, U: X \rightarrow X$ be commuting mappings satisfying

$$
d(U x, U y) \leq \mu d(S x, S y), \quad \forall x, y \in X, \mu \in[0,1) .
$$

If $U(X) \subset S(X)$ and $S$ is continuous, then $S$ and $U$ have a unique common fixed point in $X$.

\section{Remark 2.7}

(i) We shall employ both Lemmas 2.5 and 2.6 in the Banach space setting while recalling that the metric is induced by the norm. Therefore, we have $d(x, y)=\|x-y\|, x, y \in X$.

(ii) Lemma 2.6 is more general than Lemma 2.5 in the sense that we recover Lemma 2.5 from Lemma 2.6 if $S=I=$ identity operator in (6).

(iii) Several authors have generalized and extended Banach's Fixed Point Theorem [1, 2, 5] as well as Jungck's Theorem [11] in different directions. In particular, interested readers can consult the recent articles of the authors [21-23] and a host of others in the literature. 
We introduce and employ the iterative technique below:

Let $X$ be a normed space. Consider a sequence of operators $\left\{T_{i}: X \rightarrow X\right\}_{i=0}^{k}$ and the operator $S: X \rightarrow X$. For any $x_{0} \in X$, the sequence $\left\{S x_{n}\right\}_{n=0}^{\infty} \subset X$ is defined by

$$
S x_{n+1}=\alpha_{n, 0} S x_{n}+\sum_{i=1}^{k} \alpha_{n, i} T_{i} x_{n}, \sum_{i=0}^{k} \alpha_{n, i}=1, \quad n=0,1,2, \ldots,
$$

$\alpha_{n, i} \geq 0, \alpha_{n, 0} \neq 0, \alpha_{n, i} \subset[0,1]$, where $k$ is a fixed integer and $T_{0}=I=$ identity operator. The iterative process defined in (7) shall be nomenclated as Jungck-Kirk-Mann type iterative algorithm for a sequence of operators.

\section{Remark 2.8}

(i) With $T_{i}=T^{i}$ and $S=I=$ Identity operator; then, Jungck-Kirk-Mann type iterative algorithm (7) reduces to the Kirk-Mann iterative algorithm of Olatinwo [18].

(ii) If $T_{i}=T^{i}$, the algorithm (7) becomes the Jungck-Kirk-Mann iterative algorithm defined in Olatinwo [19].

(iii) The iterative processes of Kirk [12], Krasnoselskij [13], Mann [14], Picard [26] and Schaefer [33] are also some of the special cases of Jungck-Kirk-Mann type iterative algorithm.

(iv) If $k=1$ in (7), we obtain the Jungck-Mann iterative algorithm of Singh et al. [34] as a special case.

(v) Indeed, if in (7), $S=I=$ Identity operator, then we obtain the following general KirkMann type iterative algorithm: For any $x_{0} \in X$, the sequence $\left\{x_{n}\right\}_{n=0}^{\infty} \subset X$ is defined by

$$
x_{n+1}=\sum_{i=0}^{k} \alpha_{n, i} T_{i} x_{n}, \sum_{i=0}^{k} \alpha_{n, i}=1, \quad n=0,1,2, \ldots,
$$

$\alpha_{n, i} \geq 0, \alpha_{n, 0} \neq 0, \alpha_{n, i} \subset[0,1]$, where $k$ is a fixed integer and $T_{0}=I=$ identity operator. The general Kirk-Mann type iterative algorithm defined in (8) is contained in Olatinwo [17].

We shall employ both algorithms defined in (7) and (8) to obtain our results in the present article. Some examples of operators satisfying our contractive condition are given later at the end of each section.

Lemma $2.9[5,17-19]$ Let $\delta$ be a real number such that $0 \leq \delta<1$ and $\left\{\epsilon_{n}\right\}_{n=0}^{\infty}$ a sequence of positive numbers such that $\lim _{n \rightarrow \infty} \epsilon_{n}=0$. Then, for any sequence of positive numbers $\left\{u_{n}\right\}_{n=0}^{\infty}$ satisfying

$$
u_{n+1} \leq \delta u_{n}+\epsilon_{n}, \quad n=0,1,2, \ldots,
$$

we have $\lim _{n \rightarrow \infty} u_{n}=0$. 
Throughout, we shall assume that $H$ is a real Hilbert space and $\langle.,$.$\rangle an inner product on$ $H$. Also, let $\|\cdot\|$ be the norm induced by the inner product.

Sections 3 and 4 are devoted to our main results:

\section{Convergence of iterative algorithms to common fixed points}

Theorem 3.1 Let $D$ be a nonempty closed convex subset of a real Hilbert space $H$ and let the operator $S: D \rightarrow D$ be commuting with each $T_{i}: D \rightarrow D(i=1,2, \ldots, k)$ such that $S$ is continuous and $T_{i}(D) \subset S(D)$, for each $i$. Suppose that $\left\{T_{i}: D \rightarrow D\right\}_{i=1}^{k}$ is a sequence of Jungck generalized pseudo-contractive and Lipschitzian type operators with corresponding constants $r_{i}$ and $L_{i}$ such that $0<r_{i}<L_{i}<1(i=1,2, \ldots, k)$. Then:

(i) all $T_{i}(i=1,2, \ldots, k)$ and $S$ have a unique common fixed point $x^{\star} \in D$;

(ii) for each $x_{0} \in D$, the Jungck-Kirk-Mann type iterative algorithm $\left\{S x_{n}\right\}_{n=0}^{\infty}$ defined by (7) converges strongly to $x^{\star} \forall \alpha_{n, 0} \in(0,1)$ satisfying

$$
0<\alpha_{n, 0}<\frac{2\left(L^{2}-r\right)}{\left(1-2 r+L^{2}\right)}
$$

with $\max \left\{L_{i}\right\}_{i=1}^{k}=L, \max \left\{r_{i}\right\}_{i=1}^{k}=r, 0<r<L<1$.

Proof We define the operator $U$ associated to $S$ and $T_{i}$ (for each $i=1,2, \ldots, k$ ) by

$$
U(x)=\alpha_{n, 0} S x+\sum_{i=1}^{k} \alpha_{n, i} T_{i} x, \quad \forall x \in X, \quad \alpha_{n, i} \in(0,1) .
$$

Let $U$ and $S$ be commuting mappings such that $U(D) \subset S(D)$. Since $D$ is a convex subset of $H$, we have $U(D) \subset D$ for each $\alpha_{n, i} \in(0,1)$. Since $D$ is a closed subset of a Hilbert space, $D$ is a complete metric space.

We claim that $\forall x, y \in D$,

$$
\|U x-U y\| \leq \mu\|S x-S y\|, \quad \text { where } \quad \mu=\left[\left(1-2 r+L^{2}\right) \alpha_{n, 0}^{2}-2\left(L^{2}-r\right) \alpha_{n, 0}+L^{2}\right]^{\frac{1}{2}} .
$$

Since each $T_{i}(i=1,2, \ldots, k)$ is Jungck generalized pseudo-contractive, then we have

$$
\begin{gathered}
\left\langle T_{1} x-T_{1} y, S x-S y\right\rangle \leq r_{1}\|S x-S y\|^{2},\left\langle T_{2} x-T_{2} y, S x-S y\right\rangle \leq r_{2}\|S x-S y\|^{2}, \\
\ldots,\left\langle T_{k} x-T_{k} y, S x-S y\right\rangle \leq r_{k}\|S x-S y\|^{2} .
\end{gathered}
$$

Also, since each $T_{i}(i=1,2, \ldots, k)$ is Lipschitzian type, we have

$$
\begin{aligned}
\left\|T_{1} x-T_{1} y\right\| \leq L_{1}\|S x-S y\|,\left\|T_{2} x-T_{2} y\right\| & \leq L_{2}\|S x-S y\|, \ldots,\left\|T_{k} x-T_{k} y\right\| \\
& \leq L_{k}\|S x-S y\| .
\end{aligned}
$$


Let

$$
\max \left\{r_{1}, r_{2}, \ldots, r_{k}\right\}=r>0, \max \left\{L_{1}, L_{2}, \ldots, L_{k}\right\}=L, \quad r<L<1
$$

Now,

$$
\begin{aligned}
\|U x-U y\|^{2}= & \left\|\alpha_{n, 0}(S x-S y)+\sum_{i=1}^{k} \alpha_{n, i}\left(T_{i} x-T_{i} y\right)\right\|^{2} \\
= & \alpha_{n, 0}^{2}\|S x-S y\|^{2}+2 \alpha_{n, 0}\left\langle\sum_{i=1}^{k} \alpha_{n, i}\left(T_{i} x-T_{i} y\right), S x-S y\right\rangle \\
& +\left\|\sum_{i=1}^{k} \alpha_{n, i}\left(T_{i} x-T_{i} y\right)\right\|^{2} .
\end{aligned}
$$

Since each $T_{i}(i=1,2, \ldots, k)$ is Lipschitzian type, we obtain by (13) and the second part of (14) that

$$
\begin{aligned}
\left\|\sum_{i=1}^{k} \alpha_{n, i}\left(T_{i} x-T_{i} y\right)\right\| & =\left\|\alpha_{n, 1}\left(T_{1} x-T_{1} y\right)+\alpha_{n, 2}\left(T_{2} x-T_{2} y\right)+\cdots+\alpha_{n, k}\left(T_{k} x-T_{k} y\right)\right\| \\
& \leq \alpha_{n, 1}\left\|T_{1} x-T_{1} y\right\|+\alpha_{n, 2}\left\|T_{2} x-T_{2} y\right\|+\cdots+\alpha_{n, k}\left\|T_{k} x-T_{k} y\right\| \\
& \leq \alpha_{n, 1} L_{1}\|S x-S y\|+\alpha_{n, 2} L_{2}\|S x-S y\|+\cdots+\alpha_{n, k} L_{k}\|S x-S y\| \\
& \leq\left(\alpha_{n, 1}+\alpha_{n, 2}+\cdots+\alpha_{n, k}\right) L\|S x-S y\|=L\left(\sum_{i=1}^{k} \alpha_{n, i}\right)\|S x-S y\| \\
& =\left(1-\alpha_{n, 0}\right) L\|S x-S y\|, \text { since } \sum_{i=0}^{k} \alpha_{n, i}=1,
\end{aligned}
$$

from which we have

$$
\left\|\sum_{i=1}^{k} \alpha_{n, i}\left(T_{i} x-T_{i} y\right)\right\|^{2} \leq\left(1-\alpha_{n, 0}\right)^{2} L^{2}\|S x-S y\|^{2} .
$$

Also, since each $T_{i}(i=1,2, \ldots, k)$ is Jungck generalized pseudo-contractive, we obtain by (12) and the first part of (14) that 


$$
\begin{aligned}
&\left\langle\sum_{i=1}^{k} \alpha_{n, i}\left(T_{i} x-T_{i} y\right), S x-S y\right\rangle=\left\langle\alpha_{n, 1}\left(T_{1} x-T_{1} y\right)+\sum_{i=2}^{k} \alpha_{n, i}\left(T_{i} x-T_{i} y\right), S x-S y\right\rangle \\
&=\alpha_{n, 1}\left\langle T_{1} x-T_{1} y, S x-S y\right\rangle+\left\langle\sum_{i=2}^{k} \alpha_{n, i}\left(T_{i} x-T_{i} y\right), S x-S y\right\rangle \\
&=\alpha_{n, 1}\left\langle T_{1} x-T_{1} y, S x-S y\right\rangle \\
& \quad+\left\langle\alpha_{n, 2}\left(T_{2} x-T_{2} y\right)+\sum_{i=3}^{k} \alpha_{n, i}\left(T_{i} x-T_{i} y\right), S x-S y\right\rangle \\
&=\alpha_{n, 1}\left\langle T_{1} x-T_{1} y, S x-S y\right\rangle+\alpha_{n, 2}\left\langle T_{2} x-T_{2} y, S x-S y\right\rangle \\
& \quad+\left\langle\sum_{i=3}^{k} \alpha_{n, i}\left(T_{i} x-T_{i} y\right), S x-S y\right\rangle \\
&=\alpha_{n, 1}\left\langle T_{1} x-T_{1} y, S x-S y\right\rangle+\alpha_{n, 2}\left\langle T_{2} x-T_{2} y, S x-S y\right\rangle \\
& \quad+\cdots+\alpha_{n, k}\left\langle T_{k} x-T_{k} y, S x-S y\right\rangle \\
& \leq\left(\sum_{i=1}^{k} \alpha_{n, i} r_{i}\right)\|S x-S y\|^{2} \leq\left(\sum_{i=1}^{k} \alpha_{n, i}\right) r\|S x-S y\|^{2} \\
&=\left(1-\alpha_{n, 0}\right) r\|S x-S y\|^{2}, \text { since } \sum_{i=0}^{k} \alpha_{n, i}=1 .
\end{aligned}
$$

Using (16) and (17) in (15) yield

$$
\|U x-U y\|^{2} \leq\left[\left(1-2 r+L^{2}\right) \alpha_{n, 0}^{2}-2\left(L^{2}-r\right) \alpha_{n, 0}+L^{2}\right]\|S x-S y\|^{2} .
$$

Thus, showing from (18) that

$$
\|U x-U y\| \leq \mu\|S x-S y\|, \quad \text { with } \quad \mu=\left[\left(1-2 r+L^{2}\right) \alpha_{n, 0}^{2}-2\left(L^{2}-r\right) \alpha_{n, 0}+L^{2}\right]^{\frac{1}{2}} .
$$

We now prove that $\mu \in(0,1)$ :

We have that $1-2 r+L^{2}=(1-r)^{2}+L^{2}-r^{2}>0$, since $0<r<1, r<L$.

Now,

$$
\begin{aligned}
\mu^{2} & =\left(1-2 r+L^{2}\right) \alpha_{n, 0}^{2}-2\left(L^{2}-r\right) \alpha_{n, 0}+L^{2} \\
& =\left(1-2 r+L^{2}\right)\left[\alpha_{n, 0}^{2}-\frac{2\left(L^{2}-r\right)}{1-2 r+L^{2}} \alpha_{n, 0}+\frac{L^{2}}{1-2 r+L^{2}}\right] \\
& =\left(1-2 r+L^{2}\right)\left(\alpha_{n, 0}-\frac{L^{2}-r}{1-2 r+L^{2}}\right)^{2}+\frac{L^{2}-r^{2}}{1-2 r+L^{2}} .
\end{aligned}
$$

We now have the following cases by using Condition (9): 
Case 1 Given that $\alpha_{n, 0}>0$. Then, using (19) gives

$$
\begin{aligned}
\mu^{2} & =\left(1-2 r+L^{2}\right)\left(\alpha_{n, 0}-\frac{L^{2}-r}{1-2 r+L^{2}}\right)^{2}+\frac{L^{2}-r^{2}}{1-2 r+L^{2}} \\
& >\frac{\left(L^{2}-r\right)^{2}}{1-2 r+L^{2}}+\frac{L^{2}-r^{2}}{1-2 r+L^{2}}=L^{2}>0, \text { since } r<1, r<L .
\end{aligned}
$$

So, we have $\mu^{2}>0$, from which either $\mu<0$, or, $\mu>0$. Since $\mu$ must be positive, we therefore, choose $\mu>0$.

Case 2 Given that $\lambda<\frac{2\left(L^{2}-r\right)}{\left(1-2 r+L^{2}\right)}$. Then, we have by using (19) again that

$$
\begin{gathered}
\mu^{2}=\left(1-2 r+L^{2}\right)\left(\alpha_{n, 0}-\frac{L^{2}-r}{1-2 r+L^{2}}\right)^{2}+\frac{L^{2}-r^{2}}{1-2 r+L^{2}} \\
<\frac{\left(L^{2}-r\right)^{2}}{1-2 r+L^{2}}+\frac{L^{2}-r^{2}}{1-2 r+L^{2}}=L^{2}<1, \text { since } r<L<1 .
\end{gathered}
$$

from which we have $\mu^{2}<1$. That is, $(\mu+1)(\mu-1)<0$. Thus, $-1<\mu<1$. Thus, combining the ranges of validity of $\mu$ in both cases yields $0<\mu<1$. That is, we have $\mu \in(0,1)$. Therefore, this completes the proof of the second part of the claim given in (11).

Next, since $\mu \in(0,1), U$ and $S$ commute with $U(D) \subset S(D)$ and that $S$ is continuous, then we have by Lemma 2.6 (Jungck's fixed point theorem) again that $U$ and $S$ satisfying Inequality (11) have a unique common fixed point. Again, by extension, $S$ and each $T_{i}$ have a unique common fixed point since $S$ and each $T_{i}$ commute, $S$ is continuous and $T_{i}(D) \subset S(D)$ (for each $\left.i=1,2, \ldots, k\right)$.

Indeed, the unique common fixed point of $S$ and $U$ is computed by the Jungck-KirkMann's iterative algorithm $\left\{S x_{n}\right\}_{n=0}^{\infty}$ defined by

$$
S x_{n+1}=U x_{n}=\alpha_{n, 0} S x_{n}+\sum_{i=1}^{k} \alpha_{n, i} T_{i} x_{n}, \quad n=0,1,2, \ldots,
$$

Hence, the unique common fixed point of $S$ and $T$ is simultaneously and automatically computed too.

Therefore, we now prove (ii) of the result using Jungck-Kirk-Mann type iterative algorithm $\left\{S x_{n}\right\}_{n=0}^{\infty} \subset D$ as follows: We have that if $x^{\star}$ is a common fixed point of $S$ and $\left\{T_{i}\right\}_{i=1}^{k}$, then $S x^{\star}=T_{i} x^{\star}=x^{\star}$ (for each $\left.i=1,2, \ldots, k\right)$. So,

$$
\begin{aligned}
\left\|S x_{n+1}-x^{\star}\right\|^{2}= & \left\|\alpha_{n, 0} S x_{n}+\sum_{i=1}^{k} \alpha_{n, i} T_{i} x_{n}-\alpha_{n, 0} S x^{\star}-\sum_{i=1}^{k} \alpha_{n, i} T_{i} x^{\star}\right\|^{2} \\
= & \left\|\alpha_{n, 0}\left(S x_{n}-S x^{\star}\right)+\sum_{i=1}^{k} \alpha_{n, i}\left(T_{i} x_{n}-T_{i} x^{\star}\right)\right\|^{2} \\
= & \alpha_{n, 0}^{2}\left\|S x_{n}-S x^{\star}\right\|^{2}+2 \alpha_{n, 0}\left\langle\sum_{i=1}^{k} \alpha_{n, i}\left(T_{i} x_{n}-T_{i} x^{\star}\right), S x_{n}-S x^{\star}\right\rangle \\
& +\left\|\sum_{i=1}^{k} \alpha_{n, i}\left(T_{i} x_{n}-T_{i} x^{\star}\right)\right\|^{2}
\end{aligned}
$$


Again, using (16) and (17) in (20) yield

$$
\begin{aligned}
\left\|S x_{n+1}-x^{\star}\right\| \leq \mu\left\|S x_{n}-x^{\star}\right\| & \leq \mu^{2}\left\|S x_{n-1}-x^{\star}\right\| \\
& \leq \cdots \leq \mu^{n+1}\left\|S x_{0}-x^{\star}\right\| \rightarrow 0 \text { as } n \rightarrow \infty,
\end{aligned}
$$

where $\mu=\left[\left(1-2 r+L^{2}\right) \alpha_{n, 0}^{2}-2\left(L^{2}-r\right) \alpha_{n, 0}+L^{2}\right]^{\frac{1}{2}}, \mu \in(0,1)$.

Hence, the Jungck-Kirk-Mann type iterative algorithm $\left\{S x_{n}\right\}_{n=0}^{\infty}$ converges strongly to the unique common fixed point $x^{\star}$ of $S$ and $\left\{T_{i}\right\}_{i=1}^{k}$.

Theorem 3.2 Let D be a nonempty closed convex subset of a real Hilbert space $H$ and let the operator $S: D \rightarrow D$ be commuting with each $T_{i}: D \rightarrow D(i=1,2, \ldots, k)$ such that $S$ is continuous and $T_{i}(D) \subset S(D)$, for each $i$. Suppose that $\left\{T_{i}: D \rightarrow D\right\}_{i=1}^{k}$ is a sequence of Jungck generalized pseudo-contractive and nonexpansive type operators with corresponding constants $r_{i}$ such that $0<r_{i}<1(i=1,2, \ldots, k)$. Then:

(i) all $_{i}(i=1,2, \ldots, k)$ and $S$ have a unique common fixed point $x^{\star} \in D$;

(ii) for each $x_{0} \in D$, the Jungck-Kirk-Mann type iterative algorithm $\left\{S x_{n}\right\}_{n=0}^{\infty}$ defined by (7) converges strongly to $x^{\star} \forall \alpha_{n, 0} \in(0,1)$ with $\max \left\{r_{i}\right\}_{i=1}^{k}=r, 0<r<1$.

Proof The operator $U$ is as defined in (19) in Theorem 3.1. The line of argument in the proof of Theorem 3.2 is similar to that of Theorem 3.1 except that in the present case $L=1$ since each $T_{i}$ is nonexpansive type in addition to being Jungck-generalized pseudo-contractive. Therefore, we have

$$
\|U x-U y\|^{2} \leq\left[2(1-r) \alpha_{n, 0}^{2}-2(1-r) \alpha_{n, 0}+1\right]\|S x-S y\|^{2},
$$

It follows from (30) that

$$
\|U x-U y\| \leq\left[2(1-r) \alpha_{n, 0}^{2}-2(1-r) \alpha_{n, 0}+1\right]^{\frac{1}{2}}\|S x-S y\|=\eta\|S x-S y\|,
$$

with

$$
\eta=\left[2(1-r) \alpha_{n, 0}^{2}-2(1-r) \alpha_{n, 0}+1\right]^{\frac{1}{2}}
$$

We now show that $\eta \in(0,1)$ using the fact that $\alpha_{n, 0} \in(0,1)$ as follows: So, we have

$$
\eta^{2}=2(1-r) \alpha_{n, 0}^{2}-2(1-r) \alpha_{n, 0}+1=2(1-r)\left[\left(\alpha_{n, 0}-\frac{1}{2}\right)^{2}+\frac{1+r}{4(1-r)}\right]
$$

Also, we have the following cases:

Case 1 Given that $\alpha_{n, 0}>0$. Then, using (23) gives

$$
\eta^{2}=2(1-r)\left[\left(\alpha_{n, 0}-\frac{1}{2}\right)^{2}+\frac{1+r}{4(1-r)}\right]>0 \text {, since } 0<r<1 .
$$

Since $\eta^{2}>0$ as in Case 1 of Theorem 2.1, then we conclude that $\eta>0$.

Case 2 Given that $\alpha_{n, 0}<1$. Then, we have by using (23) again that 


$$
\eta^{2}=2(1-r)\left[\left(\alpha_{n, 0}-\frac{1}{2}\right)^{2}+\frac{1+r}{4(1-r)}\right]<\frac{(1-r)}{2}+\frac{1+r}{2}=1,
$$

from which we obtain $\eta^{2}<1$. That is, $(\eta+1)(\eta-1)<0$, which is the same inequality at which we arrived in Case 2 of Theorem 3.1.

Hence, we obtain $\eta \in(0,1)$.

Therefore, with $\eta \in(0,1)$, we have by the application of Lemma 2.6 again that $S$ and $T_{i}$ (for each $i=1,2, \ldots, k$ ) have a unique common fixed point. The unique common fixed point of $S$ and $T_{i}$ (for each $i=1,2, \ldots, k$ ) is computed by the Jungck-Kirk-Mann type iterative algorithm as in Theorem 3.1.

We also prove (ii) of the result using Jungck-Kirk-Mann type iterative algorithm $\left\{S x_{n}\right\}_{n=0}^{\infty} \subset D$ as follows: we have that if $x^{\star}$ is a common fixed point of $S$ and each $T_{i}$, then $S x^{\star}=T_{i} x^{\star}=x^{\star}$. Then, as in Theorem 3.1, we have that

$$
\begin{aligned}
\left\|S x_{n+1}-x^{\star}\right\|^{2}= & \alpha_{n, 0}^{2}\left\|S x_{n}-S x^{\star}\right\|^{2}+2 \alpha_{n, 0}\left\langle\sum_{i=1}^{k} \alpha_{n, i}\left(T_{i} x_{n}-T_{i} x^{\star}\right), S x_{n}-S x^{\star}\right\rangle \\
& +\left\|\sum_{i=1}^{k} \alpha_{n, i}\left(T_{i} x_{n}-T_{i} x^{\star}\right)\right\|^{2} \\
\leq & {\left[2(1-r) \alpha_{n, 0}^{2}-2(1-r) \alpha_{n, 0}+1\right]\left\|S x_{n}-x^{\star}\right\|^{2}, }
\end{aligned}
$$

from which we obtain that

$$
\left\|S x_{n+1}-x^{\star}\right\| \leq \eta\left\|S x_{n}-x^{\star}\right\|, \eta=\left[2(1-r) \alpha_{n, 0}^{2}-2(1-r) \alpha_{n, 0}+1\right]^{\frac{1}{2}},
$$

$\eta \in(0,1)$.

We therefore have from (24) that

$$
\begin{aligned}
\left\|S x_{n+1}-x^{\star}\right\| \leq \eta\left\|S x_{n}-x^{\star}\right\| & \leq \eta^{2}\left\|S x_{n-1}-x^{\star}\right\| \\
& \leq \cdots \leq \eta^{n+1}\left\|S x_{0}-x^{\star}\right\| \rightarrow 0 \text { as } n \rightarrow \infty,
\end{aligned}
$$

since $\eta \in(0,1)$. Hence, the Jungck-Kirk-Mann iterative algorithm $\left\{S x_{n}\right\}_{n=0}^{\infty}$ converges strongly to $x^{\star}$ which is the unique common fixed point of $S$ and $T_{i}$ (for each $i=1,2, \ldots, k$ ).

Theorem 3.3 Let $D$ be a nonempty closed convex subset of a real Hilbert space $H$ and let $\left\{T_{i}: D \rightarrow D\right\}_{i=1}^{k}$ be a sequence of generalized pseudo-contractive and Lipschitzian operators with corresponding constants $q_{i}$ and $L_{i}$ such that $0<q_{i}<L_{i}<1$. Then:

(i) all $T_{i}(i=1,2, \ldots, k)$ have a unique common fixed point $x^{\star} \in D$;

(ii) for each $x_{0} \in D$, the general Kirk-Mann type iterative algorithm $\left\{x_{n}\right\}_{n=0}^{\infty}$ defined by (8) converges strongly to $x^{\star}, \forall \alpha_{n, 0} \in(0,1)$ satisfying

$0<\alpha_{n, 0}<\frac{2\left(L^{2}-q\right)}{\left(1-2 q+L^{2}\right)}$, with $\max \left\{L_{i}\right\}_{i=1}^{k}=L, \max \left\{q_{i}\right\}_{i=1}^{k}=q, \quad 0<q<L<1$. 
Proof When $S=I=$ Identity operator in the statement of Theorem 3.1 and its proof, then we obtain the proof of Theorem 3.3. However, Lemma 2.5 (Banach's fixed point theorem $[1,2,5])$ is invoked here rather than Jungck's fixed point theorem [11].

Remark 3.4 Theorem 3.1 and Theorem 3.3 are generalizations of Theorem 2.1 of Verma [42], while Theorem 3.2 is also an extension of Theorem 3.1 of Verma [42] (which is itself Theorem 3.6 of Berinde [5]).

Example 3.5 Let

$$
H=\mathbb{R}
$$

(the real line) be endowed with the Euclidean inner product and norm. Let $X=\left[0, \frac{3}{4}\right] \subset H$ and $S, T_{i}: X \rightarrow X(i=1,2)$ mappings defined by

$$
T_{1} x=\frac{1}{4} x^{4}, \quad T_{2} x=\frac{1}{8} x^{8}, \quad \forall x \in X \quad \text { and } \quad S x=x^{2}, \quad \forall x \in X .
$$

Then, $\left\{T_{i}\right\}_{i=1}^{2}$ is a sequence of Jungck generalized pseudo-contractive and Lipschitzian type mappings with $T_{i}(X) \subseteq S(X)(i=1,2)$. The common fixed point set of $S$ and $\left\{T_{i}\right\}_{i=1}^{2}$ is given by $F\left(S \cap\left\{T_{i}\right\}_{i=1}^{2}\right)=\{0\}$.

Solution The fixed point set of $S$ is given by $F(S)=X=\left[0, \frac{3}{4}\right]$, while the common fixed point set of $\left\{T_{i}\right\}_{i=1}^{2}$ is given by $F\left(\cap_{i=1}^{2} T_{i}\right)=\{0\}$. Then, the common fixed point set of $S$ and $\left\{T_{i}\right\}_{i=1}^{2}$ is $F\left(S \cap\left\{T_{i}\right\}_{i=1}^{2}\right)=\{0\}$.

The mapping $S$ is continuous in $X$.

Now, we show that $T_{i}$ (for each $i=1,2$ ) is a Lipschitzian type as follows: With $T_{1} x=\frac{1}{4} x^{4}$, we have

$$
\begin{aligned}
\left\|T_{1} x-T_{1} y\right\|=\left|T_{1} x-T_{1} y\right|=\frac{1}{4}\left|x^{4}-y^{4}\right| & =\frac{1}{4}\left|x^{2}+y^{2}\right| \cdot\left|x^{2}-y^{2}\right|=\frac{1}{4}\left|x^{2}+y^{2}\right| \cdot|S x-S y| \\
& \leq \frac{9}{32}\|S x-S y\|, \quad \text { with } \quad L_{1}=\frac{9}{32} .
\end{aligned}
$$

Again, with $T_{2} x=\frac{1}{8} x^{8}$, we have

$$
\begin{aligned}
\left\|T_{2} x-T_{2} y\right\|=\left|T_{2} x-T_{2} y\right|=\frac{1}{8}\left|x^{8}-y^{8}\right| & =\frac{1}{8}\left|x^{4}+y^{4}\right| \cdot\left|x^{2}+y^{2}\right| \cdot\left|x^{2}-y^{2}\right| \\
& \leq \frac{729}{8192}\|S x-S y\|, \quad \text { with } \quad L_{2}=\frac{729}{8192},
\end{aligned}
$$

and thus, showing that $T_{i}$ (for each $i=1,2$ ) is Lipschitzian type.

We now show that $T_{i}$ (for each $i=1,2$ ) is a Jungck generalized pseudo-contractive mapping as follows: We have $T_{1}(X)=\left[0, \frac{81}{2^{10}}\right] \subset\left[0, \frac{9}{16}\right]=S(X), T_{2}(X)=\left[0, \frac{3^{8}}{2^{19}}\right] \subset\left[0, \frac{9}{16}\right]=S(X)$.

Case 1 (i) With $T_{1} x=\frac{1}{4} x^{4}, \forall x \in X$, let $u=0, v=\frac{1}{4}$ and $r=\frac{1}{2}$. So, we have $T_{1} u=0, T_{1} v=\frac{1}{4^{5}}, S u=0, S v=\frac{1}{4^{2}}$ and $T_{1}(X) \subset S(X)$. Then, we obtain 


$$
\begin{aligned}
r^{2}\|S u-S v\|^{2}+\left\|T_{1} u-T_{1} v-r(S u-S v)\right\|^{2} & =\left(\frac{1}{2}\right)^{2}\left|-\frac{1}{4^{2}}\right|^{2}+\left|-\frac{1}{4^{5}}-\frac{1}{2}\left(-\frac{1}{4^{2}}\right)\right|^{2} \\
& =\frac{1}{2^{10}}+\left(\frac{1}{2^{5}}-\frac{1}{2^{10}}\right)^{2} \\
& =\frac{2^{11}-63}{2^{20}}>\left\|T_{1} u-T_{1} v\right\|^{2}=\frac{1}{2^{20}},
\end{aligned}
$$

which implies that $T_{1}$ is Jungck generalized pseudo-contractive.

Case 1 (ii) With $T_{1} x=\frac{1}{4} x^{4}, \forall x \in X$, let $u=0, v=\frac{3}{4} \quad$ and $\quad r=\frac{1}{2}$. So, $T_{1} u=0, T_{1} v=\frac{3^{4}}{4^{5}}, S u=0, S v=\left(\frac{3}{4}\right)^{2}$ and $T_{1}(X) \subset S(X)$. Then,

$$
\begin{aligned}
r^{2}\|S u-S v\|^{2}+\left\|T_{1} u-T_{1} v-r(S u-S v)\right\|^{2} & =\left(\frac{1}{2}\right)^{2}\left|-\left(\frac{3}{4}\right)^{2}\right|^{2}+\left|-\frac{3^{4}}{4^{5}}-\frac{1}{2}\left(-\left(\frac{3}{4}\right)^{2}\right)\right|^{2} \\
& =\frac{3^{4}}{2^{10}}+\left(\frac{3^{2}}{2^{5}}-\frac{3^{4}}{2^{10}}\right)^{2} \\
& =\frac{81\left(2^{11}-495\right)}{2^{20}}>\left\|T_{1} u-T_{1} v\right\|^{2}=\frac{3^{8}}{2^{20}},
\end{aligned}
$$

which again implies that $T_{1}$ is Jungck generalized pseudo-contractive.

Case 2 (i) With $T_{2} x=\frac{1}{8} x^{8}, \forall x \in X$, let $u=0, v=\frac{1}{4}$ and $r=\frac{1}{2}$. So, we have $T_{1} u=0, T_{1} v=\frac{1}{2^{19}}, S u=0, S v \stackrel{8}{=} \frac{1}{4^{2}}$ and $T_{2}(X) \subset S(X)$. Then, we obtain

$$
\begin{aligned}
r^{2}\|S u-S v\|^{2}+\left\|T_{1} u-T_{1} v-r(S u-S v)\right\|^{2} & =\left(\frac{1}{2}\right)^{2}\left|-\frac{1}{4^{2}}\right|^{2}+\left|-\frac{1}{2^{19}}-\frac{1}{2}\left(-\frac{1}{4^{2}}\right)\right|^{2} \\
& =\frac{1}{2^{10}}+\left(\frac{1}{2^{5}}-\frac{1}{2^{19}}\right)^{2} \\
& =\frac{1+2^{29}-2^{15}}{2^{38}}>\left\|T_{1} u-T_{1} v\right\|^{2}=\frac{1}{2^{38}},
\end{aligned}
$$

which implies that $T_{2}$ is Jungck generalized pseudo-contractive.

Case 2 (ii) With $T_{2} x=\frac{1}{8} x^{8}, \forall x \in X$, let $u=0, v=\frac{3}{4} \quad$ and $\quad r=\frac{1}{2}$. So, $T_{1} u=0, T_{1} v=\frac{3^{8}}{2^{19}}, S u=0, S v=\left(\frac{3}{4}\right)^{2}$ and $T_{2}(X) \subset S(X)$. Then,

$$
\begin{aligned}
r^{2}\|S u-S v\|^{2}+\left\|T_{2} u-T_{2} v-r(S u-S v)\right\|^{2} & =\left(\frac{1}{2}\right)^{2}\left|-\left(\frac{3}{4}\right)^{2}\right|^{2}+\left|-\frac{3^{8}}{2^{19}}-\frac{1}{2}\left(-\left(\frac{3}{4}\right)^{2}\right)\right|^{2} \\
& =\frac{3^{4}}{2^{10}}+\left(\frac{3^{2}}{2^{5}}-\frac{3^{8}}{2^{19}}\right)^{2} \\
& =\frac{81\left(3^{12}+2^{29}-3^{6} \times 2^{15}\right)}{2^{38}}>\left\|T_{2} u-T_{2} v\right\|^{2}=\frac{3^{16}}{2^{38}},
\end{aligned}
$$

which again implies that $T_{2}$ is Jungck generalized pseudo-contractive.

Thus, $\left\{T_{i}\right\}_{i=1}^{2}$ is a sequence of Jungck generalized pseudo-contractive and Lipschitzian type mappings. 


\section{Stability results for Jungck-Kirk-Mann type iterative algorithm and general Kirk-Mann type iterative algorithm}

The rest of this article is devoted to the study of stability of Jungck-Kirk-Mann type iterative algorithm and general Kirk-Mann type iterative algorithm. We now state the following definition:

Suppose that $(X, d)$ is a complete metric space, $\left\{T_{i}: X \rightarrow X\right\}_{i=1}^{n}$ a sequence of mappings and $S: X \rightarrow X$ a mapping. Denote by $F\left(S \cap T_{i}\right)$ the set of all the common fixed points of $S$ and $\left\{T_{i}\right\}_{i=1}^{n}$, which is given by

$$
F\left(S \cap T_{i}\right)=\left\{x^{\star} \in X \mid T_{i} x^{\star}=S x^{\star}=x^{\star}\right\}, \text { for each } i .
$$

Definition 4.1 Let $S: X \rightarrow X$ and $\left\{T_{i}: X \rightarrow X\right\}_{i=1}^{k}, T_{i}(X) \subset S(X)$, be mappings defined on a complete metric space $(X, d)$. Let $x^{\star}$ be a common fixed point of $S$ and $T_{i}$, that is,

$$
T_{i} x^{\star}=S x^{\star}=x^{\star}, \quad \text { or, } \quad x^{\star} \in F\left(S \cap T_{i}\right) .
$$

For any $x_{0} \in X$ (initial approximation), let the sequence $\left\{S x_{n}\right\}_{n=0}^{\infty} \subset X$ generated by the iterative algorithm

$$
S x_{n+1}=h\left(\left\{T_{i}\right\}_{i=1}^{k}, x_{n}\right), \quad n=0,1,2, \ldots,
$$

converge to $x^{\star}$, where $h$ is some function. Let $\left\{S y_{n}\right\}_{n=0}^{\infty} \subset X$ be an arbitrary sequence and set

$$
\epsilon_{n}=d\left(S y_{n+1}, h\left(\left\{T_{i}\right\}_{i=1}^{k}, y_{n}\right)\right), \quad(n=0,1,2, \ldots) .
$$

Then, the iterative procedure $(\Delta \star)$ will be called $\left(S,\left\{T_{i}\right\}_{i=1}^{k}\right)$-stable, or, stable with respect to $S$ and the sequence of mappings $\left\{T_{i}\right\}_{i=1}^{k}$ whenever $\lim _{n \rightarrow \infty} \epsilon_{n}=0$ if and only if $\lim _{n \rightarrow \infty} S y_{n}=x^{\star}$.

That is, $(\Delta \star)$ is $\left(S,\left\{T_{i}\right\}_{i=1}^{k}\right)$-stable whenever

$$
\lim _{n \rightarrow \infty} \epsilon_{n}=0 \Longleftrightarrow \lim _{n \rightarrow \infty} S y_{n}=x^{\star} .
$$

\section{Remark 4.2}

(i) Since metric is induced by norm, then we have that

$$
d(x, y)=\|x-y\|, \quad x, y \in X,
$$

whenever we are working in the normed linear space setting.

(ii) Definition 4.1 reduces to that of Olatinwo [17] if in $(\Delta \star), S=I=$ identity mapping.

(iii) Again, Definition 4.1 becomes that of [10] if in $(\Delta \star), k=1$ and $S=I$ (identity mapping).

(iv) Furthermore, Definition 4.1 is independent of the Definition 2.1 of stability given in [34]. 
The following are the stability results for the iterative algorithms aforementioned at the beginning of this section:

Theorem 4.3 Let $D$ be a nonempty closed convex subset of a real Hilbert space H. Suppose that $S: D \rightarrow D$ is continuous and $\left\{T_{i}: D \rightarrow D\right\}_{i=1}^{k}$ is a sequence of Jungck generalized pseudo-contractive and Lipschitzian type operators with corresponding constants $r_{i}, L_{i}$ such that $0<r_{i}<L_{i}<1(i=1,2, \ldots, k)$ and $T_{i}(D) \subset S(D)$, for each $i$. For each $x_{0} \in D$, let $\left\{S x_{n}\right\}_{n=0}^{\infty}$ be the Jungck-Kirk-Mann type iterative algorithm defined by (7). Then, $\left\{S x_{n}\right\}_{n=0}^{\infty}$ is $\left(S,\left\{T_{i}\right\}_{i=1}^{k}\right)$-stable, $\forall \alpha_{n, 0} \in(0,1)$ satisfying

$$
0<\alpha_{n, 0}<\frac{2\left(L^{2}-r\right)}{\left(1-2 r+L^{2}\right)} \text {, with } \max \left\{L_{i}\right\}_{i=1}^{k}=L, \max \left\{r_{i}\right\}_{i=1}^{k}=r, \quad 0<r<L<1 .
$$

Proof Now, if $x^{\star}$ is a common fixed point of $S$ and $\left\{T_{i}\right\}_{i=1}^{k}$, then $S x^{\star}=T_{i} x^{\star}=x^{\star}$ (for each $i=1,2, \ldots, k)$. So, let $\left\{S y_{n}\right\}_{n=0}^{\infty} \subset D$ and define

$$
\epsilon_{n}=\left\|S y_{n+1}-\alpha_{n, 0} S y_{n}-\sum_{i=1}^{k} \alpha_{n, i} T_{i} y_{n}\right\| \quad(n=0,1,2, \ldots) .
$$

Assume $\lim _{n \rightarrow \infty} \epsilon_{n}=0$. Then, we shall establish that $\lim _{n \rightarrow \infty} S y_{n}=x^{\star}$ by using the conditions satisfied by the operators, Lemma 1.11 as well as the triangle inequality as follows:

$$
\begin{aligned}
\left\|S y_{n+1}-x^{\star}\right\| \leq & \left\|S y_{n+1}-\left(\alpha_{n, 0} S y_{n}+\sum_{i=1}^{k} \alpha_{n, i} T_{i} y_{n}\right)\right\| \\
& +\left\|\alpha_{n, 0} S y_{n}+\sum_{i=1}^{k} \alpha_{n, i} T_{i} y_{n}-\left(\alpha_{n, 0} S x^{\star}+\sum_{i=1}^{k} \alpha_{n, i} T_{i} x^{\star}\right)\right\| \\
= & \left\|\alpha_{n, 0}\left(S y_{n}-S x^{\star}\right)+\sum_{i=1}^{k} \alpha_{n, i}\left(T_{i} y_{n}-T_{i} x^{\star}\right)\right\|+\epsilon_{n} .
\end{aligned}
$$

Now, using (16) and (17) again (with $x=y_{n}, y=x^{\star}$ ) yield

$$
\begin{aligned}
\left\|\alpha_{n, 0}\left(S y_{n}-S x^{\star}\right)+\sum_{i=1}^{k} \alpha_{n, i}\left(T_{i} y_{n}-T_{i} x^{\star}\right)\right\|^{2} & =\alpha_{n, 0}^{2}\left\|S y_{n}-S x^{\star}\right\|^{2}+\left\|\sum_{i=1}^{k} \alpha_{n, i}\left(T_{i} y_{n}-T_{i} x^{\star}\right)\right\|^{2} \\
& +2 \alpha_{n, 0}\left\langle\sum_{i=1}^{k} \alpha_{n, i}\left(T_{i} y_{n}-T_{i} x^{\star}\right), S y_{n}-S x^{\star}\right\rangle \\
\leq & \mu^{2}\left\|S y_{n}-S x^{\star}\right\|^{2},
\end{aligned}
$$

with $\mu=\left[\left(1-2 r+L^{2}\right) \alpha_{n, 0}^{2}-2\left(L^{2}-r\right) \alpha_{n, 0}+L^{2}\right]^{\frac{1}{2}}, \mu \in(0,1)$.

Therefore, it follows from (26) that

$$
\left\|\alpha_{n, 0}\left(S y_{n}-S x^{\star}\right)+\sum_{i=1}^{k} \alpha_{n, i}\left(T_{i} y_{n}-T_{i} x^{\star}\right)\right\| \leq \mu\left\|S y_{n}-S x^{\star}\right\| .
$$


Using (27) in (25) gives

$$
\left\|S y_{n+1}-x^{\star}\right\| \leq \mu\left\|S y_{n}-x^{\star}\right\|+\epsilon_{n} .
$$

Since $\mu \in(0,1)$, then applying Lemma 2.9 in (28) gives $\lim _{n \rightarrow \infty} S y_{n+1}=x^{\star}$.

Conversely, let $\lim _{n \rightarrow \infty} S y_{n}=x^{\star}$. Then, we shall establish that $\lim _{n \rightarrow \infty} \epsilon_{n}=0$ by using (27) and the triangle inequality as follows:

$$
\begin{aligned}
\epsilon_{n} & \leq\left\|S y_{n+1}-x^{\star}\right\|+\left\|\alpha_{n, 0} S x^{\star}+\sum_{i=1}^{k} \alpha_{n, i} T_{i} x^{\star}-\left(\alpha_{n, 0} S y_{n}+\sum_{i=1}^{k} \alpha_{n, i} T_{i} y_{n}\right)\right\| \\
& =\left\|S y_{n+1}-x^{\star}\right\|+\left\|\alpha_{n, 0}\left(S x^{\star}-S y_{n}\right)+\sum_{i=1}^{k} \alpha_{n, i}\left(T_{i} x^{\star}-T_{i} y_{n}\right)\right\| \\
& \leq\left\|S y_{n+1}-x^{\star}\right\|+\mu\left\|S y_{n}-x^{\star}\right\| \rightarrow 0 \text { as } n \rightarrow \infty .
\end{aligned}
$$

Hence, the Jungck-Kirk-Mann type iterative algorithm is $T_{i}$-stable (for each $i$ ).

The following is a stability result for the general Kirk-Mann type iterative algorithm for a sequence of generalized pseudo-contractive and Lipschitzian operators:

Theorem 4.4 Let $D$ be a nonempty closed convex subset of a real Hilbert space $H$ and let $\left\{T_{i}: D \rightarrow D\right\}_{i=1}^{k}$ be a sequence of generalized pseudo-contractive and Lipschitzian operators with corresponding constants $q_{i}$ and $L_{i}$ such that $0<q_{i}<L_{i}<1$. For each $x_{0} \in D$, let $\left\{x_{n}\right\}_{n=0}^{\infty}$ be the general Kirk-Mann type iterative algorithm defined by (8). Then, $\left\{x_{n}\right\}_{n=0}^{\infty}$ is $\left\{T_{i}\right\}_{i=1}^{k}$-stable, $\forall \alpha_{n, 0} \in(0,1)$ satisfying

$$
0<\alpha_{n, 0}<\frac{2\left(L^{2}-q\right)}{\left(1-2 q+L^{2}\right)} \text {, with } \max \left\{L_{i}\right\}_{i=1}^{k}=L, \max \left\{q_{i}\right\}_{i=1}^{k}=q, \quad 0<q<L<1 .
$$

Proof When $S=I=$ Identity operator in the statement of Theorem 4.3 and its proof, then we obtain the proof of Theorem 4.4.

Remark 4.5 Theorems 4.3 and 4.4 can be generalized and extended for some other fixed point iterative algorithms. Also, the results can be further improved in the current space as well as in some other spaces.

We refer to Example 3.5 again to investigate the stability of the Jungck-Kirk-Mann type iterative algorithm defined in (7) as follows:

Example 4.6 Let

$$
H=\mathbb{R}
$$

(the real line) be endowed with the Euclidean inner product and norm. Let $X=\left[0, \frac{3}{4}\right] \subset H$ and let $S, T_{i}: X \rightarrow X(i=1,2, \ldots, k)$ be mappings such that $S x=x^{2}, \forall x \in X$. The common fixed point set of $S$ and $\left\{T_{i}\right\}_{i=1}^{k}$ is given by $F\left(S \cap\left\{T_{i}\right\}_{i=1}^{k}\right)=\{0\}$. Define an arbitrary sequence $\left\{y_{n}\right\}_{n=0}^{\infty} \subset X$ by $y_{n}=\frac{1}{n^{2}+1}(n=0,1,2, \ldots)$. Then, the Jungck-Kirk-Mann type iterative algorithm 
defined in (7) is $\left(S,\left\{T_{i}\right\}_{i=1}^{k}\right)$-stable, with $\alpha_{n, 0}=\frac{1}{4}$, and $\left\{T_{i}\right\}_{i=1}^{k}$ is a sequence of Jungck generalized pseudo-contractive and Lipschitzian type mappings such that $\max \left\{L_{i}\right\}_{i=1}^{k}=L=\frac{9}{32}, \max \left\{r_{i}\right\}_{i=1}^{k}=r=\frac{1}{4}$, with $T_{i}(X) \subseteq S(X)(i=1,2, \ldots, k)$.

Solution Recall that $F\left(S \cap\left\{T_{i}\right\}_{i=1}^{k}\right)=\left\{x^{\star}\right\}=\{0\}$. We observe that $S$ is a continuous mapping in $X$. Again, the Jungck-Kirk-Mann type iterative algorithm is the following: for any $x_{0} \in X$, the sequence $\left\{S x_{n}\right\}_{n=0}^{\infty} \subset X$ is defined by

$$
S x_{n+1}=\alpha_{n, 0} S x_{n}+\sum_{i=1}^{k} \alpha_{n, i} T_{i} x_{n}, \alpha_{n, 0} \neq 0, \sum_{i=0}^{k} \alpha_{n, i}=1, \quad(n=0,1,2, \ldots) .
$$

Since $\alpha_{n, 0}=\frac{1}{4}=r, L=\frac{9}{32}$, we have that

$$
\mu=\left[\left(1-2 r+L^{2}\right) \alpha_{n, 0}^{2}-2\left(L^{2}-r\right) \alpha_{n, 0}+L^{2}\right]^{\frac{1}{2}}=\left(\frac{205.5625}{1024}\right)^{\frac{1}{2}} \in(0,1) .
$$

With $y_{n}=\frac{1}{n^{2}+1}, n=0,1,2, \ldots$, then, the arbitrary sequence $\left\{S y_{n}\right\}_{n=0}^{\infty}$ becomes $S y_{n}=\frac{1}{\left(n^{2}+1\right)^{2}}$.

Assume $\lim _{n \rightarrow \infty} \epsilon_{n}=0$. Then, we have from (28) that

$$
\begin{aligned}
\left\|S y_{n+1}-x^{\star}\right\|=\left|S y_{n+1}-x^{\star}\right|=\left|S y_{n+1}\right| & \leq \mu \| S y_{n}-x^{\star} \mid+\epsilon_{n} \\
& =\mu\left|S y_{n}\right|+\epsilon_{n}=\frac{\mu}{\left(n^{2}+1\right)^{2}}+\epsilon_{n} \rightarrow 0 \quad \text { as } \quad n \rightarrow \infty,
\end{aligned}
$$

showing that $\lim _{n \rightarrow \infty} S y_{n}=x^{\star}=0$.

In a converse manner, let $\lim _{n \rightarrow \infty} S y_{n}=x^{\star}$. Then, we have from the second part of the proof of Theorem 3.3 that

$$
\begin{aligned}
\epsilon_{n} \leq\left\|S y_{n+1}-x^{\star}\right\|+\mu\left\|S y_{n}-x^{\star}\right\| & =\left|S y_{n+1}\right|+\mu\left|S y_{n}\right| \\
& =\frac{1}{\left(n^{2}+2 n+2\right)^{2}}+\frac{\mu}{\left(n^{2}+1\right)^{2}} \rightarrow 0 \text { as } n \rightarrow \infty .
\end{aligned}
$$

Hence, the Jungck-Kirk-Mann type iterative algorithm is $\left(S,\left\{T_{i}\right\}_{i=1}^{k}\right)$-stable.

Remark 4.7 Indeed, the significance of this article is not only limited to generalizations and extensions of some results in the Hilbert space, or, Banach space setting, but also to introduce more classes of operators with which experts in the fixed point theory can now define and solve new problems. Also, it is our ardent hope that the applicability of our new operators by various experts will begin to emerge in due course.

\section{Conclusion}

In Theorem 3.1, we have introduced a Jungck-Kirk-Mann type iterative algorithm to establish the existence and uniqueness of a common fixed point for an operator $S$ and a sequence of operators $\left\{T_{i}: D \rightarrow D\right\}_{i=1}^{k}$ in a real Hilbert space $H$, where $\left\{T_{i}\right\}_{i=1}^{k}$ is a sequence of Jungck generalized pseudo-contractive and Lipschitzian type operators. The strong convergence of the Jungck-Kirk-Mann type iterative algorithm to the unique common fixed point of $S$ and $\left\{T_{i}\right\}_{i=1}^{k}$ is also proved under the same assumptions on each $T_{i}$. A similar result is also established in Theorem 3.2 for the same iterative algorithm 
when $\left\{T_{i}\right\}_{i=1}^{k}$ is a sequence of Jungck generalized pseudo-contractive and nonexpansive type operators.

Moreover, in Theorem 3.3, we have established similar result for a general Kirk-Mann type iterative algorithm when $\left\{T_{i}\right\}_{i=1}^{k}$ is a sequence of generalized pseudo-contractive and Lipschitzian operators.

In addition, two stability results have been established for both iterative algorithms employed in this article. Theorem 4.3 has illustrated the new stability notion given in Definition 4.1, while Theorem 4.4 improves, generalizes and extends the stability results contained in [17]. It has been shown in Theorem 4.3 that the Jungck-Kirk-Mann type iterative algorithm is $\left(S,\left\{T_{i}\right\}_{i=1}^{k}\right)$-stable, while the general Kirk-Mann type iterative algorithm is also shown to be $\left\{T_{i}\right\}_{i=1}^{k}$-stable in Theorem 4.4.

Supplementary Information The online version contains supplementary material available at https://doi. org/10.1007/s12215-021-00653-3.

Open Access This article is licensed under a Creative Commons Attribution 4.0 International License, which permits use, sharing, adaptation, distribution and reproduction in any medium or format, as long as you give appropriate credit to the original author(s) and the source, provide a link to the Creative Commons licence, and indicate if changes were made. The images or other third party material in this article are included in the article's Creative Commons licence, unless indicated otherwise in a credit line to the material. If material is not included in the article's Creative Commons licence and your intended use is not permitted by statutory regulation or exceeds the permitted use, you will need to obtain permission directly from the copyright holder. To view a copy of this licence, visit http://creativecommons.org/licenses/by/4.0/.

\section{References}

1. Agarwal, R.P., O'Regan, D., Sahu, D.R.: Fixed Point Theory for Lipschitzian-type Mappings with Applications-Topological Fixed Point Theory, vol. 6. Springer, Berlin (2009)

2. Banach, S.: Sur les operations dans les ensembles abstraits et leur applications aux equations integrales. Fund. Math. 3, 133-181 (1922)

3. Berinde, V.: Approximating fixed points of Lipschitzian pseudocontractions. In: Mathematics and Mathematics Education, Bethlehem, 2000, pp. 73-81. World Sci. Publishing, River Edge (2002)

4. Berinde, V.: Comparing Krasnoselkij and Mann iterative methods for Lipschitzian generalized pseudo-contractions. In: Proc. Int. Conference on Fixed Point Theory and Applications, Valencia (Spain), pp. 15-26 (2003)

5. Berinde, V.: Iterative Approximation of Fixed Points. Springer, Berlin (2007)

6. Borwein, J., Reich, S., Shafrir, I.: Krasnoselskii-Mann iterations in normed spaces. Can. Math. Bull. 35, 21-28 (1992)

7. Browder, F.E., Petryshyn, W.V.: The solution by iteration of nonlinear functional equations in Banach space. Bull. Am. Math. Soc. 72, 571-575 (1966)

8. Browder, F.E., Petryshyn, W.V.: Construction of fixed points of nonlinear mappings in Hilbert spaces. J. Math. Anal. Appl. 20, 197-228 (1967)

9. Haghi, R.H., Postolache, M., Rezapour, Sh.: On T-stability of the Picard iteration for generalized $\varphi$ -contraction mappings. Abstr. Appl. Anal. Vol. 2012, ID: 658971

10. Harder, A.M., Hicks, T.L.: Stability results for fixed point iteration procedures. Math. Japonica 33(5), 693-706 (1988)

11. Jungck, G.: Commuting mappings and fixed points. Am. Math. Mon. 83, 261-263 (1976)

12. Kirk, W.A.: On Successive Approximations for nonexpansive Mappings in Banach Spaces. Glasgow Math. J. 12, 6-9 (1971)

13. Krasnoselskij, M.A.: Two remarks on the method of successive approximations. Uspekhi Mat. Nauk. 10(1)(63), 123-127 (1955)

14. Mann, W.R.: Mean value methods in iteration. Proc. Am. Math. Soc. 44, 506-510 (1953) 
15. Olaleru, J.O., Akewe, H.: On the convergence of Jungck-type iterative schemes for generalized contractive-like operators. Fasciculi Mathematici 45, 87-98 (2010)

16. Olatinwo, M.O.: A result on segmenting Jungck-Mann iterates. Acta Univ. laki. Olomuc. Fac. Rer. Nat. Mathematica 47, 115-119 (2008)

17. Olatinwo, M.O.: Some stability results for two hybrid fixed point iterative algorithms of Kirk-Ishikawa and Kirk-Mann type. J. Adv. Math. Stud. 1(1-2), 87-96 (2008)

18. Olatinwo, M.O.: Some stability results for two hybrid fixed point iterative algorithms in normed linear Space. Mat. Vesn. 61(4), 247-256 (2009)

19. Olatinwo, M.O.: Stability results for Jungck-Kirk-Mann and Jungck-Kirk hybrid iterative algorithms. Anal. Theory Appl. 29(1), 12-20 (2013)

20. Olatinwo, M.O.: Some results on stability for Schaefer and Mann fixed point iterative algorithms in Hilbert spaces. Trans. Math. Program. Appl. 2(8), 17-24 (2014)

21. Olatinwo, M.O.: Some common fixed point theorems of Jungck-Fisher type for A-type mappings. J. Adv. Math. Stud. 12(3), 333-340 (2019)

22. Olatinwo, M.O., Omidire, O.J.: Fixed point theorems of Akram-Banach type. Nonlinear Anal. Forum 21(2), 55-64 (2016)

23. Olatinwo, M.O., Postolache, M.: Stability results for Jungck-type iterative processes in convex metric spaces. Appl. Math. Comput. 218(12), 6727-6732 (2012)

24. Osilike, M.O., Udomene, A.: Short proofs of stability results for fixed point iteration procedures for a class of contractive-type mappings. Indian J. Pure Appl. Math. 30(12), 1229-1234 (1999)

25. Ostrowski, A.M.: The round-off stability of iterations. Z. Angew. Math. Mech. 47, 77-81 (1967)

26. Picard, E.: Memoire sur la Theorie des Equations aux Derivees partielles et la Methode des Approximations Successives. J. Math. Pures et Appl. 6, 145-210 (1890)

27. Reich, S.: Fixed point iterations of nonexpansive mappings. Pac. J. Math. 60(2), 195-198 (1975)

28. Reich, S.: Weak convergence theorems for nonexpansive mappings in Banach spaces. J. Math. Appl. 67, 274-276 (1979)

29. Reich, S., Thong, D.V., Cholamjiak, P., Long, L.V.: Inertial projection-type methods for solving pseudomonotone variational inequality problems in Hilbert space. Numer. Algorithm. 87, 1-23 (2021)

30. Rhoades, B.E.: Fixed point theorems and stability results for fixed point iteration procedures. Indian J. Pure Appl. Math. 21(1), 1-9 (1990)

31. Rhoades, B.E.: Some fixed point iteration procedures. Int. J. Math. Math. Sci. 14(1), 1-16 (1991)

32. Rhoades, B.E.: Fixed point theorems and stability results for fixed point iteration procedures II. Indian J. Pure Appl. Math. 24(11), 691-703 (1993)

33. Schaefer, H.: Uber die methode Sukzessiver approximationen. Jahresber. Deutsch. Math. Verein. 59, 131-140 (1957)

34. Singh, S.L., Bhatnagar, C., Mishra, S.N.: Stability of Jungck-type iterative procedures. Int. J. Math. Math. Sci. 19, 3035-3043 (2005)

35. Suantai, S., Kankam, K., Cholamjiak, P.: A projected forward-backward algorithm for constrained minimization with applications to image inpainting. Mathematics 9(8), 890 (2021). https://doi.org/ 10.3390/math9080890

36. Suantai, S., Noor, M.A., Kankam, K., Cholamjiak, P.: Novel forward-backward algorithms for optimization and applications to compressive sensing and image inpainting. Adv. Differ. Equ. (2021). https://doi.org/10.1186/s13662-021-03422-9

37. Thong, D.V., Cholamjiak, P.: Strong convergence of a forward-backward splitting method with a new step size for solving monotone inclusions. Comput. Appl. Math. 38, 94 (2019). https://doi.org/ 10.1007/s40314-019-0855-z

38. Uşurelu, G.I.: Split feasibility handled by a single-projection three-step iteration with comparative analysis. J. Nonlinear Convex Anal. 22(3), 544-558 (2021)

39. Uşurelu, G.I., Postolache, M.: Convergence analysis for a three-step Thakur iteration for Suzukitype nonexpansive mappings with visualization. Symmetry-Basel 11(12), Art. No. 1441 (2019)

40. Uşurelu, G.I., Bejenaru, A., Postolache, M.: Operators with property (E) as concerns numerical analysis and visualization. Numer. Funct. Anal. Optim. 41, 1398-1411 (2020)

41. Uşurelu, G.I., Bejenaru, A., Postolache, M.: Newton-like methods and polynomiographic visualization of modified Thakur processes. Int. J. Comput. Math. https://doi.org/10.1080/00207160.2020. 1802017

42. Verma, R.U.: A fixed point theorem involving Lipschitzian generalized pseudo-contractions. Proc. Roy. Irish Acad. Soct. A 97(1), 83-86 (1997)

43. Verma, R.U.: An approximation procedure for fixed points of strongly Lipschitz operators. Portugal. Math. 54(4), 461-465 (1997) 
44. Verma, R.U.: On fixed points of Lipschitzian strongly Lipschitz operators. Math. Sci. Res. HotLine 1(7), 20-26 (1997)

45. Verma, R.U.: An iterative algorithm on fixed points of relaxed Lipschitz operators. J. Appl. Math. Stoch. Anal. 10(2), 187-189 (1997)

46. Verma, R.U.: Mann type algorithms for the fixed points of Lipschitzian strongly Lipschitz operators. Math. Sci. Res. Hot-Line 2(10), 7-12 (1998)

Publisher's Note Springer Nature remains neutral with regard to jurisdictional claims in published maps and institutional affiliations. 